\title{
ISSUES AND CHALLENGES FACING THE TEACHING OF CLOUD COMPUTING FOR THE FIRST TIME
}

\author{
Azad Ali, Indiana University of Pennsylvania, azad.ali@iup.edu \\ David T. Smith, Indiana University of Pennsylvania, david.smith@iup.edu \\ Thomas A. Leslie, Indiana University of Pennsylvania, thomas.leslie@iup.edu
}

\begin{abstract}
This paper discusses the issues and challenges facing the teaching of courses on the cloud platform (henceforth "cloud computing") for the first time. It illustrates the experience of a faculty who recently started teaching a course in cloud computing and the specific issues and challenges he faced that are unique to this new emerging platform. This faculty thought that it will be helpful to share this experience.

The paper begins by giving a literature review to explain the various factors involved in the teaching of cloud computing. In particular, it focuses on the differences between the traditional way of teaching computer subjects using platforms within the institution (henceforth we have termed "on premise computing") and using the cloud. Then the paper shifts the focus to explain the experience of this faculty in dealing with this new platform. Paper includes some suggestions on dealing with these issues and a summary of the paper is listed at the end.
\end{abstract}

Keywords: Cloud Computing, Virtualization, Teaching on the Cloud

\section{INTRODUCTION}

Cloud computing is a new computing platform that is gaining popularity among various organizations and different academic institutions (Alharthi et al., 2017, Bruin \& Floridi, 2017, Han, 2010). Companies large and small are moving their computing platforms from existing on premise computing to the new in the cloud platform to gain various advantages, such as virtualization and scalability. Similarly, academic institutions are introducing courses and proposing programs that prepare the students to work on this new platform (Gholami, et al., 2016).

The transition of teaching on the cloud is not a straight forward process, instead it is often faced with challenges that need to be addressed along the transition (Fahmideh \& Beydoun, 2018, Hu, Chen \& We, 2016). Shedding light on this transition process to the cloud along with discussing the challenges faced in the transition will be of benefit to many who teach in the same field of study, and is therefore the objective of this paper.

A faculty at an Information Systems department at a university located in Western Pennsylvania recently started teaching cloud computing for the first time. This faculty faced different challenges when teaching this new cloud platform that are unique to the selection of this platform. This faculty thought it will be helpful to share his experience. But in order to understand the issues, some introductory information is necessary. Thus, this paper starts by giving a literature review on cloud computing. The remainder of this paper is divided into the following sections:

- The paper starts by summarizing a literature review about cloud computing, meaning, development, and the most common terms exchanged to understand this platform.

- The next section explains the unique challenges that are faced when teaching on the cloud. The challenges are unique because they are not faced when teaching other non-cloud courses.

- The last section explains about the case of this paper, about the faculty that taught on the cloud for the first time, and how he addressed the challenges that he faced. 


\begin{abstract}
ABOUT CLOUD COMPUTING
Although the term "cloud computing" is widely exchanged and repeated in the news, in textbooks and on the Internet, the exact meaning of the term is not totally agreed. This section gives background information about cloud computing, some definitions and the main characteristics. It differentiates between public and private cloud and introduces other background information.
\end{abstract}

\title{
Cloud Computing Definitions
}

There have been many definitions introduced to define cloud computing, Sultan (2010) noted that there are at least 22 different definitions. This variation in definitions stem from the fact that it is a new field of study, and the multiple factors involved. Some of the definitions are brief and touches on just one aspect of it, yet other definitions provide broader descriptions and introduce factors that touch on several different related aspects. Among the comprehensive definitions we reviewed is the following offered by the National Institute of Standards and Technology (NIST)) which defines cloud computing as the following:

Cloud computing is a model for enabling ubiquitous, convenient, on-demand network access to a shared pool of configurable computing resources (e.g., networks, servers, storage, applications, and services) that can be rapidly provisioned and released with minimal management effort or service provider interaction. (Mell \& Grance, 2011, p. 2).

The definition above explains about a wide range of computing resources (storage, applications, networks) that are configured on demand and provided to others. Another definition offered by Alharthi et al. (2017) touches on the term of "cloud computing" from the perspective of migration to external resources and described it as a "Process of moving applications, data, servers, networks from in-premises to the cloud data centers (p. 666)".

The term "data centers" that is included in Alharthi's definition needs further explanation since it forms the foundation on which cloud computing researchers base their work (Nzanywayingoma and Yang 2017). Data centers typically host a vast repository of data on many volumes of data storage devices. The role of the data centers then extends beyond data storage to include processing, applications, networking, management and the distribution of data of an enterprise. These data centers may be scattered around the world and the computing resources are then accessible on demand by individuals and organizations connecting to the cloud.

Armbrust et al. (2010) simplified the definition of cloud computing and explained it in terms of the applications delivered over the Internet and the hardware and software provided externally in remote locations named data centers. Khaiiali (2017) on the other hand noted that "Cloud computing is a model of computing through which services are commoditized and in the same way as utilities such as water, electricity, gas and telephony" (p. 52). The interesting point of Kaiiali definition is the similarities in consumption of utilities it suggest that everyone uses.

While there are many different definitions and approaches to explain cloud computing, the essential definition can be best expressed in terms of its main characteristics.

\section{Main Characteristics of Cloud Computing}

Mell and Grace (2011) summarized the characteristics that can distinguish cloud computing from traditional on premise computing:

- On demand self-service. This means that the services provided on the cloud are demanded (or consumed) by the others.

- Broad network access. Cloud computing places (or data centers) offer broad network access to services that enable their subscribers to connect to them through standard networks.

- $\quad$ Resource pooling. Resource services can be pooled between multiple organizations that can be purchased and be limited to those specified in the resource pooling.

- Rapid elasticity. Resource services available to a particular organization can range from simple to comprehensive, and can be re-provisioned with relative ease.

- Measured service. The utilization of a service is measured in some ways such as hours accessed, space provided, number of users allowed and so forth. 
A quick review of the above shows that the word "service" is repeated more often when discussing cloud computing in the working on the cloud. This can be explained that cloud companies offer services and subscribers and organizations consume (subscribe) to these services. But the services vary and thus categorized and explained in several different service models of cloud computing.

\section{Service Models in Cloud Computing}

Services provided in cloud computing can fall into three different service models (categories) as explained below:

- Software as a Service (SaaS), Barton (2015) described SaaS as "a way of licensing and delivering software by using a subscription model. Subscription can be paid monthly or yearly basis or through special licensing programs". With SaaS, software is located in the cloud and users access it through the Internet.

- Platform as a Service (PaaS). Collier and Shahan (2015) described PaaS as "computing features in which applications are deployed into virtual machines of server types referred as web roles and web worker roles" (p. 46).

- Infrastructure as a Service (IaaS). Tulloch (2013) described PaaS as "customer develops and deploys applications for a specific applications architectures. The hoster provides application runtime, storage and integration needed to run the customer needed applications and is responsible for keeping the environment up and running, operating systems update and customer data safe".

The major difference between cloud computing and traditional computing is about the location where the service is provided. Services in cloud computing are not saved on resources (e.g. hard disks and network drives) of the organization. Instead, they are saved on remote locations called the cloud. Access to these remote locations are often limited to one company, while in other cases, the access can leased and subscribed to. This particular difference in access is what distinguishes private and public cloud.

\section{Private and Public Cloud}

Private cloud are platforms created by different companies and the resources are available for their own use. In other words, the cloud services for these companies are not available to the public, instead they are only available to individual given access granted by this company (typically employees of the company that owns the cloud). Companies that provide private clouds for their computing services are on the rise. More and more companies are switching to the cloud to provide services for their own business.

Public cloud on the other hand is explained as computing services offered by third-party providers over the public Internet, making them available to anyone who wants to use or purchase those services (Black, 2017). Public cloud services could be offered for free or sold on-demand, allowing customers to pay only per usage for applications, CPU cycles, storage, or bandwidth they consume (Moskalenko, 2017). Harvey (2017) listed four public cloud providers that are dominant in this field:

- $\quad$ Amazon Web Services

- $\quad$ Microsoft Azure

- $\quad$ IBM Cloud

- $\quad$ Google Cloud Platform

In this paper, we will focus on public cloud services and in particular Microsoft Azure, because it is the platform that we used for our course.

\section{Microsoft Azure}

Microsoft Azure is a cloud computing platform that provides Platform as a Service (PaaS) to the public. The web site www.wahtisup.com provides the following definition for Microsoft Azure:

Microsoft Azure, formerly known as Windows Azure, is Microsoft's public cloud computing platform. It provides a range of cloud services, including those for compute, analytics, storage and networking. Users can pick and choose from these services to develop and scale new applications, or run existing applications, in the public cloud.

The interface to the cloud services is through Azure portal providing a group of interrelated services to customers on demand. In other words, users of Microsoft Azure login to the portal and from there can choose different services. Collier and Shahan (2015) grouped Azure services into the following categories: 


\section{Issues in Information Systems \\ Volume 19, Issue 4, pp. 187-195, 2018}

- $\quad$ Azure Web sites and Azure Cloud Services

- $\quad$ Azure Virtual Machines

- $\quad$ Azure Storage

- $\quad$ Azure Virtual networks

- $\quad$ Azure Databases

- $\quad$ Azure Active Directory

- Azure Management Tools

Connecting to Azure is done through the Azure portal. Although the Azure portal is customizable and can change from individual to another, they all have same general characteristics. Azure starts by creating resource groups and then resources. Then from there, services can be added to the resources. A screen capture of Azure portal is shown in the figure 1 below.

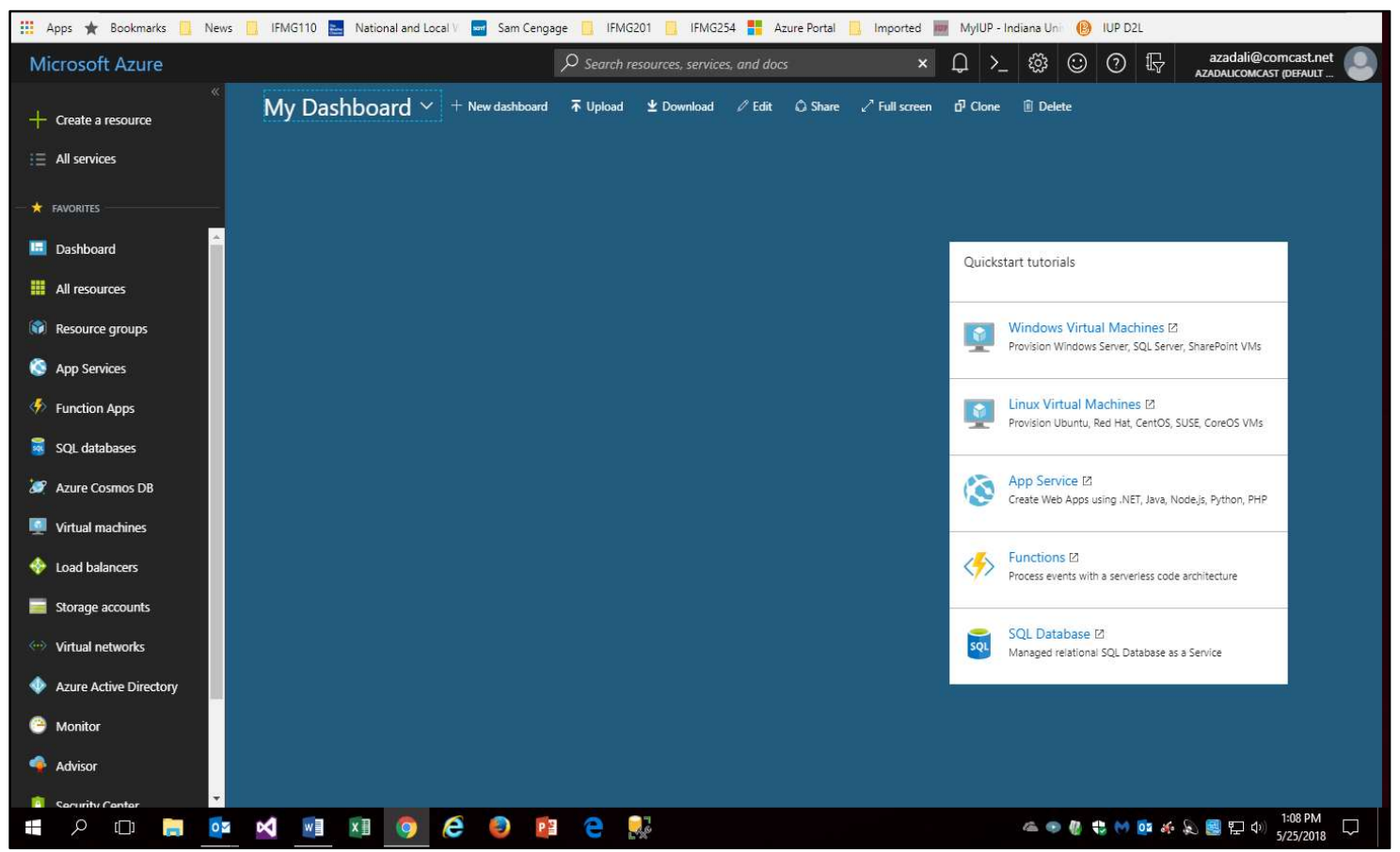

Figure 1. Azure Portal

\section{Subscription to Azure Services}

Access to using Azure is done mainly through the subscriptions, that is individuals or organizations subscribe to Microsoft Azure and are given access based on their subscription levels. Billing and paying for the services are handled differently in Azure subscriptions. Collier and Shahan (2015) listed six subscription management and billing for Microsoft Azure:

- $\quad$ Free trial

- $\quad$ MSDN subscription

- $\quad$ BizSpark accounts

- $\quad$ Pay-as-you-go

- $\quad$ Enterprise agreement

The subscriptions vary in terms of prices paid, level of services offered, bandwidth available for connection and other related factors. Microsoft also offers within the enterprise agreement another type of subscription that enables development tools to be available to students. This is called Microsoft Imagine Agreement where select tools are available to all students from different colleges and universities. We visited the web site for this imagine (https://imagine.microsoft.com/en-us ) and browsed through the different pages. We found their program benefits page that talks about 
Microsoft Imagine Standard and Microsoft Imagine Premium. These are offered at different prices, but none include all Microsoft products, instead the cover some of them.

\section{CLOUD COMPUTING - DIFFERENCE IN TEACHING}

There are a few factors that differ between teaching in a regular setting (henceforth we call it "traditional setting") of the classroom. We list some of what we found through our review.

\section{Lack of Students Text Books}

Jablonka \& Johansson (2010) suggested that textbooks can form a window into the classroom practice. In other words, it gives a view of what is going on in the classroom (Newton \& Newton, 2006). Richards (2012) suggested that textbooks can provide a number of advantages, among them include the following:

- Provide structure and syllabus for the course

- Help standardize instructions

- Maintain quality

- Provide a variety of learning resources

The level at which textbooks are followed may be another topic, but it cannot be denied that textbooks can help planning a course as if gives a systematic approach to content of a course and is a source of lectures, exercises, exams and other activities that goes in the classroom. Ali and Wibowo (2016) suggested that textbooks can also include tutorial approach to learning by giving, examples and tutorials that aid in learning.

Writers often talk about two categories of textbooks: those written for students and those written for experienced or professionals in the field. Through our research for textbooks for students, we found that student textbooks are distinguished from other textbooks because they provide more learning tools than the others. In looking at student textbooks in Information Technology (IT), we often found they included these helpful tools:

- Case illustration

- End of chapter review questions

- Examples of hands-on project

- Tutorial exercises

- Short and long exercises

- Screen captures with various annotations

- Chapter overview

- Charts, figure and diagrams

- Chapter hints and others

In a new field of study, textbooks can play an important role in learning. Giving case examples, illustrations, practical applications and other tools can play a bigger part in the learning process. This is simply true because the students do not have a previous framework to base their knowledge on. In other words, they do not have examples that happened in the past to compare the concepts to.

In the case of Information Technology field, we found that giving examples, visual illustrations, and exercises are crucial factors in learning for students attending courses at colleges. Professional textbooks, on the other hand, are often lacking these essential tools for learning. Instead professional texts primarily provide cursory examples that often rely on past experience to fully comprehend.

In our search for textbooks to teach cloud computing (or Azure), we could not find student textbooks. Most of the textbooks we found are published by Microsoft Press which tend often to provide coverage that favors the presentation of the advantages of using the platform and does not give concrete examples of their use. They also explain it from the perspective that the services run perfectly - without any problems or hitches. They do not give examples or illustrations of the problems generated form the use of the product and how resolve them. So to put it simple, the textbooks that we found do not have the helpful materials that are typically available in student textbooks that we listed above. Instead the textbooks go directly to the work of the platform, it briefly describes each and gets into the steps of completion without mentioning a hitches or points of difficulty in the platform. 


\section{The Subscription Model for Accessing Resources}

Collier and Shahan (2015) stated that access to cloud resources on Azure are available only through subscriptions. So no subscription means no service. The most suitable form of subscription for beginners is the pay-as-you-go. There is two potential issues with this subscription:

- The students are required to give credit card number so they pay for it now and secure future payments. This is a sensitive issue because many see the risk of giving credit card numbers and thus are hesitant to give them out.

- The pay-as-you-go means students who spend more time working on the cloud pay a higher price for their extra work

Both of the above points may post a challenge of implementation in the course and they are not practiced the same way in courses that do not teach cloud computing. First in terms of giving credit card number, it is true that students give credit card numbers when they purchase textbooks, but this is not the same. Textbooks are paid for once in the course, the students know how much they pay and they are done with it. In the case of pay-as-you-go prices could be different where some students pay more, others do not pay the less. So convincing the students of paying under this pay-as-you-go is a challenge.

\section{The Access to the Cloud Platforms}

As noted before, the main difference between cloud computing and on premise computing is the location of the resources that are accessed by the faculty, staff and students. In on premise computing, the resources are located on-site, whether local or remote. In other words, access to these computing resources are regulated by the rules and regulations that the organizations set for themselves. Typical rules include for example are setting login procedures, setting quota for space allocation, email regulations, file transfer protocols and many others.

In cloud computing, the resources are not located internally. Instead, the resources are located on computers that do not belong to the university itself. These resources are subscribed to the university. This creates an extra layer of interface between the user and resources that are located in data centers across the world. This means the following:

- Users will face an extra layer of log in and associated passwords and helping hints.

- When they try to access resources, they have to follow the rules set by public cloud.

- Since resources are subscribed, users have to follow the rules set by the terms of subscription, in particular the amount of connection time and pace allocated for each service.

- With more layers of interface and more regulations, there is a greater possibility of problems occurring at different levels of working on the cloud. In other words, students will be facing more errors at several levels of access.

This subscription model has limitations that makes it difficult to manage the classroom environment. For one subscriptions expire which can impeded continuity. It may set conditions for the number of users thereby limiting the times at which students can connect. Furthermore, it may set limitations in terms of setting the accounts and of type of software that are accessed, number of users to share and others.

In the case of on premise computing, users do not same have limitations on number of times logged in or number of times spent accessing the resources. Instead, the students can login as many times without incurring extra charges. Once logged onto a system, all installed resources are readily available for students to use with minimal regulations. So fewer regulations means fewer problems encountered from working on the platform.

\section{CLOUD COMPUTING - TEACHING FOR FIRST TIME}

A department of Information system in Western Pennsylvania has introduced a course in virtualization and cloud computing. The faculty teaching this course faced challenges in teaching this course for the first time that were similar to what has been discussed in this paper. This section explains the specifics of problems faced this faculty when teaching cloud computing and how he dealt with each issue. 


\title{
The Issue with the Textbooks
}

Yes at the beginning we searched for textbooks that exemplified what we have been using in our other courses. In other words, we were looking for textbooks that some of the learning aids (like tutorial projects, case illustrations, end of chapter questions) to help us in planning the content of the course. We could not find textbooks about Azure that is as helpful to the students as the textbooks that we use in other courses. Instead, most of the found textbooks on Azure were professional texts published by Microsoft Press. To address this we found other helpful resources like the following:

- YouTube videos

- Microsoft channel 9 videos for developers (https://channel9.msdn.com/)

- Tutorials that are available in each platform in Azure

- $\quad$ Step by step tutorials in a web site provided by Microsoft

Although these videos were helpful, in many cases they did not work well. They often stopped short of a complete cohesive presentation and did not illustrate the problems that may occur. They are presumed to work in a perfect environment and thus do not assume any problems.

\section{Subscription to the platform}

Our university subscribes to Microsoft Imagine agreement and makes software available to our students. However, the imagine agreement that we had offered only a limited services for free and they asked for a subscription if they want access to the other services. The free services that we found in our subscription include the following:

- $\quad$ Azure SQL server

- $\quad$ Azure Web Apps

- WordPress on Azure

We worked the course so that the students work on Azure and use the free services at the beginning of the course. Then toward the end of the course, the students can subscribe and use the 30-day trial service to use additional services. In this case, the students will not be charged. However, the subscription (even though it is free) it requires giving credit card numbers. The students were not comfortable giving their credit card numbers. They also claimed that financial aid reimburse them for textbooks (or eBooks) but do not reimburse them for this kind of subscription.

\begin{abstract}
Added Interface
The added interface is explained by giving the example of Azure Web Apps and the interface with databases created using Azure services as compared to the same process in Visual Studio. When creating web applications with Visual studio, there are a few variables you need to think about. For example, the location of the database, the programming language to use, the type of databases and a few other related variables. Most likely, students and faculty do not think that if they create another database, it will cost them more.

These variables are increased in Azure platform. You have to think about resources groups, resources, service plan, server authentication and others. So added layers that will make it harder to work with. Additionally, all are connected to the level of subscription and what is available to subscribers in that subscription. So, error messages are abound when trying to create a web app and unintentionally exceeds your limits set in the subscription levels. What complicates the issue further is the ambiguous error messages that are generated from working with these platforms. You need to be an expert in the field to understand these error messages. The extra interface and the ambiguous error messages were serious issues that we had to deal with. We worked through these by stumbling through the resources and mostly through trial-and-error because the error messages were not very helpful. We also realized that Microsoft development products in general are not easy to learn and work with, but on the cloud they are even harder to work with and generate more ambiguous error messages.
\end{abstract}

\section{SUMMARY AND CONCLUDING REMARKS}

The objective that we stated at the beginning of this paper is to discuss the platform of cloud computing and the issues that appears from using it for the first time. The paper started by talking about the cloud computing in general, it focused on the literature review and the development of cloud computing as a field of study. It then focused on the course that is taught by our department and explained how we dealt with. Our concluding remarks after teaching Azure platform for this first time is two points: 


\section{Issues in Information Systems \\ Volume 19, Issue 4, pp. 187-195, 2018}

- $\quad$ First, for new entrants to teaching cloud computing, we recommend taking this topic seriously. It is big, it is very ambiguous and it is riddled with problems. So we recommend to buckle up as you start with working on Azure. Furthermore, a lot of videos and a lot of web links for Azure do not work. Also, the error messages are often not helpful in finding a remedy.

- Second, our recommendations is to continue teaching the cloud computing (and Azure) despite the many problems we faced. This is true because the market demands moving that directions so we better offer our students skills that are marketable. It seems that the marked will demand for a long time to come, graduates with skills and training on cloud computing and virtualization will be highly valued.

\section{REFERENCES}

Alharthi, A., Alassafi, M. O., Walters, R. J., \& Wills, G. B. (2017). An exploratory study for investigating the critical success factors for cloud migration in the Saudi Arabian higher education context. Telematics and Informatics, 34(2), 664-678.

Ali, A., \& Wibowo, K. (2016). Using office simulation software in teaching computer literacy using three sets of teaching/learning activities. Issues in Informing Science and Information Technology, 13, 79-88. Retrieved from http://www.informingscience.org/Publications/3470

Armbrust, M., Fox, A., Griffith, R., Joseph, A., Katz, K., Konwinski, A., Lee, G., Patterson, D., Rabkin, A., Stoica, I., \& Zaharia, M. (2020). A View of Cloud Computing. Communication of the ACM, 53(4), 50-58.

Barton, B. (2015). Microsoft Public Cloud Services Setting up your business in the cloud., Redmond, WA: Microsoft Press

Black, N. (2017). Practicing Law in the Cloud Ethically and Securely. Gpsolo, 34(3), 28-31.

Collier, M. \& Shahan, R. (2015). Fundamentals of Azure Microsoft Azure Essentials., Redmond, WA: Microsoft Press.

Fahmideh, M. \& Beydoun, G (2018). Reusing empirical knowledge during cloud computing adoption. The Journal of Systems and Software, 138, 124-157.

Gholami, M. F., Daneshgar, F. Low, G., \& Beydoun, G (2016). Cloud migration process - A survey, evaluation framework, and open challenges. The Journal of Systems and Software, 120, 31-69.

Gholami, M. F., Daneshgar, F., Beydoun, G., \& Rabhi, F. (2017). Challenges in migrating legacy software systems to the cloud - an empirical study. Information Systems, 67, 100-113.

Harvey, C. (2017). Public Cloud Computing Providers. Datamation. Retrieved from https://www.datamation.com/cloud-computing/public-cloud-providers.html.

Jablonka, E., \& Johansson, M. (2010). Using Texts and Tasks: Swedish Studies on Mathematics Textbooks. In Sriraman, B., Bergsten, C., Goodchild, S., Paldottir, G., Søndergaard, B. D., Haapasalo, L. (Eds.), The First Sourcebook on Nordic Research in Mathematics Education: Norway, Sweden, Iceland, Denmark and contributions from Finland (pp. 363-372). Charlotte, NC: Information Age Publishing.

Kaiiali, M. (2017). Designing a VM-level vertical scalability service in current cloud platforms: a new hope for wearable computers. Turkish Journal of Electrical Engineering \& Computer Sciences, 25(4), 2555-2566.

Khan, Q., \& Sultana, N. (2012). Transformative Education, School Textbooks and Gender. FWU Journal of Social Sciences, 6(2), 109-115. 


\section{Issues in Information Systems}

Volume 19, Issue 4, pp. 187-195, 2018

Liu, Y., Sun, A. Y., Nelson, K., \& Hipke, W. E. (2013). Cloud computing for integrated stochastic groundwater uncertainty analysis. International Journal of Digital Earth, 6(4), 313-337.

Mell, P., \& Grance, T. (2011). The NIST Definition of Cloud Computing. Communications of the ACM, 53(6). Retrieved from https://nvlpubs.nist.gov/nistpubs/Legacy/SP/nistspecialpublication800-145.pdf.

Moskalenko, A. (2017). Deciding If the Cloud Is Right for Your Law Practice. Gpsolo, 34(4), 60-63.

Newton, D., \& Newton, L. (2007). Could Elementary Mathematics Textbooks Help Give Attention to Reasons in the Classroom?. Educational Studies in Mathematics, 64(1), 69-84.

Nzanywayingoma, F., \& Yang, Y. (2017). Efficient Resource Management techniques in Cloud Computing Environment: Review and discussion. Telkomnika, 15(4), 1917-1933.

Richards, J.C. (2012). The Role of Textbooks in a Language Program. ProfessorJackRichards.com. Retrieved 0512-2018 from http://www.professorjackrichards.com/pdfs/role-of-textbooks.

Sultan, N. (2010). Cloud computing for education: A new dawn? International Journal of Information Management. $30,109-116$.

Tulloch, M. with the Windows Azure Team. (2013). Introducing Windows Azure for IT Professionals. Microsoft Press. Redmond, WA. 